\title{
Redesigning Generator of Landing Craft Tank 1500 DWT by Considering Technical and Economic Factors
}

\author{
Ivan Guntur Perdana ${ }^{1}$, Mohammad Rusdy Hatuwe ${ }^{2 *}$, Amir Marasabessy ${ }^{2}$ \\ ${ }^{1}$ Undergraduate Student of Naval Engineering, Faculty of Engineering, University of Pembangunan Nasional Veteran Jakarta, Jl. RS \\ Fatmawati Raya, Pondok Labu, Jakarta Selatan, 12450, Indonesia \\ ${ }^{2}$ Faculty of Engineering, University of Pembangunan Nasional Veteran Jakarta, Jakarta Indonesia
}

\begin{abstract}
The electrical system is very important thing on a ship, where the tool that functions as power to meet the electricity needs on the ship is a generator. In some cases, generator planning does not pay attention to technical and economic factors that have an impact on operations cost. The technical factor will be adjusted to the standard and regulations of the classification board, while the economic factor will be planning an efficient electrical installation and power installation system. The results obtained from this paper show the operational needs of the ship based on the condition when harbour, sailing, loading and unloading, and emergency during the day with total intermittent load of $110,6 \mathrm{KW}$ and total continuous load of 130,6 KW while the operational needs of the ship at night with total intermittent load is $98,2 \mathrm{KW}$ and total continuous load is $181,5 \mathrm{KW}$. Then it was found that the largest operational power requirement of the ship in an emergency at night was $38,5 \mathrm{KW}$ and the largest power was in the condition of the ship at night of $102,5 \mathrm{KW}$. Then we get 3 generators with 2 sets of $60 \mathrm{KW}$ power capacity and 1 set of $45 \mathrm{KW}$.
\end{abstract}

Keywords: Redesigning, Operations Cost, Landing Craft Tank

\section{Introduction}

The electrical system on the ship is an important thing in designing a ship. Generator is one of the equipment on the ship that is useful to meet all the power needs on the ship. In determining the capacity of the generator that will be used to meet the power needs of the ship, a load calculation will be carried out to determine the power capacity and changes in use for each operating condition. The goal is to find the minimum and maximum power required [1]. In some cases, Landing Craft Tank (LCT) 1500 DWT ship at Dok dan Perkapalan Kodja Bahari Jakarta Shipyard Unit III has a very large generator power so that it becomes inefficient for planning generator power requirements. Therefore, this paper aims to redesigning generator of LCT 1500 DWT by considering technical and economic factors. The technical factor in question is in accordance with the regulations that have been issued by a class, which in this case is the Indonesian Classification Bureau (BKI). Meanwhile, the economic factor in question is the efficiency of calculations regarding the determination of power installations and lighting installations. The author will calculate the load factor and the diversity factor on the ship. The load factor will be calculated conditions when harbour, sailing, loading and unloading and emergency. To calculate the generator capacity, first requires secondary power balance data from the installed pumps, then calculates all electric motors from the winch, lamps and other electrical equipment installed according to technical factors and economic factors. According to the Indonesian Classification Bureau (BKI) listed in Volume 4 Chapter 1 of 2004, all electrical equipment on board and the capacity of each equipment must be listed in the table.

\section{Generator Capacity}

The generator output power required during sea voyages must be greater than $15 \%$ of the power requirements specified in the power balance [3].

\section{Ship Electrical and Equipment}

The electrical components contained in the ship are divided into several parts, as follows:

\subsection{Electric motor and Winch}

According to (Hartono, 1988) winch or a tool commonly called a crane is a tool that needs to be able to rotate slowly to lift or extend the tros and anchors.

\footnotetext{
* Corresponding author: mohrushhat@gmail.com
} 


\subsubsection{Windlass and anchor motor power}

Included in the Indonesian Classification Bureau (BKI) volume 2 of 2018, regarding the calculation of the anchor plan, the following formula can be used to calculate the anchor plan:

$$
Z=\frac{D 2}{3}+2 \cdot h \cdot B+\frac{A}{10}
$$

where D $=$ Displacement of the ship [m3], h $=\mathrm{fb}+\mathrm{hi}$ $[\mathrm{m}], \mathrm{B}=$ Width of the ship $[\mathrm{m}], \mathrm{A}=$ Area in side view of the hull, superstructure, and deckhouse [m2]

\subsubsection{Winch and Lifeboat Electric Motor Power}

According to the book "Ship Equipment and Supplies" written by Sukarsono N.A. On page 75, the motor power of the lifeboat can use the following formula:

$$
N=\frac{W \times H}{60 \times 75 \times \eta} ;(k w)
$$

where $\mathrm{W}=$ weight of lifeboat + weight of equipment + weight of crew [kg] According to the book "ship systems and equipment" by Sukarno NA, the lifeboat and equipment weighs $2,960 \mathrm{~kg}, \mathrm{H}=$ lifeboat height [m], $=$ motor efficiency, i.e. 0,98 (for new motorcycle)

\subsubsection{Winch and Ramp Door Motor Power}

According to the book "Merchant Ship Design Hand Book Volume 5" page 58, calculating the winch drum can use the formula:

$$
l=\frac{k \cdot d \cdot L}{\pi \cdot N \cdot(D+n \cdot d)} ;(k w)
$$

where $\mathrm{k}=$ provisions for wire rope $1,2, \mathrm{~d}=$ Steel rope $3 / 4$ inch $[\mathrm{m}], \mathrm{L}=$ Length of rolled steel rope, $\mathrm{n}=$ standard roll rope maximum 7, D = Diameter of drum Then perform Hydraulic calculations based on "Maker Bosch Rexroth Hydraulics, Theory and Applications" using the formula:

$$
M=F \times r ;(N m)
$$

where $\mathrm{F}=$ Load on winch drum $9810 \mathrm{~N}, \mathrm{r}=$ radius of winch drum $[\mathrm{m}]$

Then calculate the flow rate of the electric motor using the formula:

$$
Q=\frac{v \cdot n}{(n \cdot v \%) \cdot 10^{-1}} ;(l t / \mathrm{min})
$$

Then the calculation of the power input from the electric motor will be obtained using the formula:

$$
P=\frac{Q . p}{6 . t \%} ;(k w)
$$

where $\mathrm{Q}=$ Flow rate pump $[1 \mathrm{t} / \mathrm{min}], \mathrm{p}=207$ bar (existing), $\mathrm{t}=\mathrm{v} \times \mathrm{hm}=0.85 \times 0.98=0.765$

The last step is to calculate the power output of the electric motor using the formula:

$$
P=\frac{Q \cdot p \cdot t^{\%}}{6 \times 10^{-4}} ;(k w)
$$

where $\mathrm{Q}=$ Flow rate motor $[1 \mathrm{t} / \mathrm{min}], \mathrm{p}=207 \mathrm{bar}$ (existing), $\mathrm{t}=\mathrm{v} \times \mathrm{hm}=0.85 \times 0.98=0.765$

From the calculation above, the power from the electric motor will be obtained according to the winch.

\subsubsection{Air Conditioner (AC)}

According to the book "Ship Equipment and Supplies" written by Sukarsono N.A. On page 75, the AC motor power can use the following formula:
\[ N=\frac{3.8 \times \mathrm{V}}{100} ;(k w) \]
where $\mathrm{V}=$ Room volume $[\mathrm{m} 3]$

\subsubsection{Lighting Equipment}

Determination of lighting equipment on the ship in accordance with the book "Merchant Ship Design Hand Book Volume 5" is divided into 3 parts of lighting. First, inside Lighting (a), includes interior lighting covers rooms such as rooms on the main deck, poop deck, and wheel house deck. Second, external lighting (b), includes aisles on the outside of the ship, as well as places for lowering lifeboats. Third, navigation lighting (c), includes lights used by ships when sailing, loading and unloading or leaning, and so on. So from the three parts of the lighting, the total lighting on the ship can be calculated with the following formula:

$$
\Sigma=\text { Total } a+\text { Total } b+\text { Total } c
$$

\subsubsection{Other Electrical Equipment}

The electrical equipment in question is equipment that can support the completeness of the ship, such as equipment in the kitchen (a), washing equipment (b) and navigation and communication equipment (c), the following formula is:

$$
\Sigma=\text { Total } a+\text { Total } b+\text { Total } c
$$

\subsection{Generator Power Capacity Calculation}

In this case it will be explained about the calculations in analyzing the power capacity of the generator, as follows:

\subsubsection{Load Factor}

The load factor is very important when planning the generator capacity to distribute all the electrical power requirements on the ship. The load factor is defined as the ratio of the working time of the equipment under certain conditions to the total working time under these conditions [3].

\subsubsection{Continuous Load (CL)}

Equipment that operates continuously under normal operating conditions. Here is the equation formula

$C L=$ Input $x$ Total Work $x L F ;(k w)$ where $\mathrm{CL}=$ Continous Load $[\mathrm{kw}], \mathrm{LF}=$ Load Factor, Input $=$ Data Power Balance using Open Source software

\subsubsection{Intermittent Load (IL)}

Equipment that operates intermittently (periodically) in normal sailing conditions with an indefinite period of time. Here is the equation formula:

$$
I L=\text { Input } x \text { Total Work } x L F ;(k w)
$$

where IL $=$ Intermittern Load $[\mathrm{kw}], \mathrm{LF}=$ Load Factor, Input $=$ Data Power Balance using Open Source software 


\subsubsection{Diversity Factor}

In short, the diversity factor is the operating load in determining the total load on the generator. Where the value of the diversity factor according to the Indonesian Classification Bureau (BKI) should not be below 0.5. So the author uses a reference from the energy and electricity journal STT-PLN Vol.9 No.6 regarding "Electrical System on the Frosch-class Navy Warship KRI Teluk Celukan Bawang 532" which uses 0.6 for the diversity factor value. The following is the equation formula for calculating the diversity factor, as follows:

$$
e=\frac{\text { Total IL }}{\text { Total Power }}(k w)
$$

where $\mathrm{e}=$ Diversity Factor $[\mathrm{kw}], \mathrm{IL}=$ Intermittern Load $[\mathrm{kw}]$

\subsubsection{Power Requirement}

The following is the formula for calculating the electric power capacity (power requirement), as follows:

Power Requirement $=$ Total IL $x e ;(k w)^{(14)}$ where IL $=$ intermittent load [kw], e = Diversity Factor $[\mathrm{kw}]$

\subsubsection{Total Load Power Calculation}

The following is the formula for calculating the total load power, as follows:

$$
P B=C L+e x I L ;(k w)
$$

where $\mathrm{CL}=$ Continous Load [kw], e = Diversity Factor, $\mathrm{IL}=$ Intermittern Load $[\mathrm{kw}]$

\section{Analysis}

\subsection{Main Properties}

Main properties is obtained from secondary data collection from Dok dan Perkapalan Kodja Bahari Shipyard Unit III. This data will be used as a reference for generator calculations, it can be seen in table 1 , as follows:

Table 1. Main properties of mv. alfa trans dua

\begin{tabular}{|c|c|}
\hline Parameter & Properties \\
\hline Name & MV Alfa Trans Dua \\
\hline Length Overall (LOA) & $64 \mathrm{~m}$ \\
\hline $\begin{array}{c}\text { Length Between Perpendicular } \\
\text { (LPP) }\end{array}$ & $56.7 \mathrm{~m}$ \\
\hline Breadth (B) & $14 \mathrm{~m}$ \\
\hline Height (H) & $4.5 \mathrm{~m}$ \\
\hline Draught (T) & $3 \mathrm{~m}$ \\
\hline Coefficient Block $\left(\mathrm{C}_{\mathrm{B}}\right)$ & $0.76 \mathrm{~m}$ \\
\hline Speed (Vs) & 9 knots \\
\hline Displacement & 1950 ton \\
\hline Crew & 19 Person \\
\hline
\end{tabular}

\subsection{Windlass and Anchor Power}

The first thing to do is to calculate the anchor plan, based on BKI volume 2 can use the following formula:

Where:

$$
Z=\frac{D 2}{3}+2 \cdot h \cdot B+\frac{A}{10}
$$

$h=f b+h i$

$=1.5+6.5$

$=8$

$A=$ The area of the walls of the superstructure

$=A 1+A 2+A 3+A 4$

$=96 m 2+92 m 2+15 m 2+41 m 2$

$=172 \mathrm{~m} 2$

So,

$$
\begin{aligned}
Z & =\frac{19502}{3}+2.8 .14+\frac{172}{10} \\
Z & =156+224+17.2 \\
& =397.2
\end{aligned}
$$

So from the BKI table in section 7 regarding "ship equipment" regarding anchors, anchor chains and rigging based on the $Z$ value obtained, it can be seen in table 2, as follows:

Table 2. Data on anchor, anchor chain and rope - rigging

\begin{tabular}{|c|c|}
\hline Item & $\begin{array}{c}\text { Anchor } \\
\text { Requirement }\end{array}$ \\
\hline Number of Anchor & 2 \\
\hline Weight 1 Anchor & $1140 \mathrm{~kg}$ \\
\hline Diameter 1 (d1) & $34 \mathrm{~mm}$ \\
\hline Diameter 2 (d2) & $30 \mathrm{~mm}$ \\
\hline Diameter 3 (d3) & $26 \mathrm{~mm}$ \\
\hline Tail chain length for bow anchor (1) & $385 \mathrm{~m}$ \\
\hline pull rope length & $180 \mathrm{~m}$ \\
\hline Mooring rope length & $140 \mathrm{~m}$ \\
\hline Number of mooring ropes & 4 \\
\hline
\end{tabular}

Calculation of 1, Anchor Electric Motor Power

Diameter : $34 \mathrm{~mm}$

Brand : Data

Type : DZC 12001/12002

Tensile Force $\quad: 12000 \mathrm{~kg}$

Motor Speed : $: 12 \mathrm{~m} / \mathrm{min}$

Motor Power : $22 \mathrm{~kW}$

Then, calculation of 2 and 3, Anchor Electric Motor

Power

Diameter : $: 30 \mathrm{~mm}$ and $26 \mathrm{~mm}$

Brand : Data

Type : DZC 7001/7002

Tensile Force $\quad: 7500 \mathrm{~kg}$

Motor Speed : : 13m/min

Motor Power : $15 \mathrm{~kW}$

\subsection{Winch and Lifeboat Electric Motor Power}

Based on "Ship System and Equipment" by Sukarsono, you can use the formula: 


$$
\begin{aligned}
& N=\frac{W \times H}{60 \times 75 \times \eta} ;(k w) \\
& \text { Where: } \\
& W=\text { weight of lifeboat }+ \text { weight of equipment } \\
& + \text { weight of crew; }(\mathrm{kg}) \\
& =2960+(19 \times 75) \\
& =4385
\end{aligned}
$$

So,

$$
\begin{aligned}
N & =\frac{4385 \times 0.7}{60 \times 75 \times 0.98} ;(k w) \\
& =0.7 \mathrm{HP} \\
& =0.52 \mathrm{~kW}
\end{aligned}
$$

Then obtained davit specifications for lifeboats as follows:

\begin{tabular}{|c|c|c|c|c|}
\hline NO & Type of Materials & Size & \multicolumn{2}{|c|}{ Total } \\
\hline \multirow[t]{4}{*}{1} & Stay rampdoor plate & $12 \mathrm{~mm} \times 1550 \times 4500$ & 4 & Sh \\
\hline & & $12 \mathrm{~mm} \times 4000 \times 300$ & 2 & Sh \\
\hline & & $12 \mathrm{~mm} \times 4500 \times 300$ & 2 & Sh \\
\hline & Angle profile & L $90 \times 90 \times 9 \mathrm{~mm}$ & 6 & Bar \\
\hline \multirow[t]{3}{*}{2} & Engsel & $25 \mathrm{~mm} \times 350 \times 450$ & 18 & Sh \\
\hline & & $30 \mathrm{~mm} \times 300 \times 450$ & 9 & Sh \\
\hline & Bracket & $12 \mathrm{~mm} \times 150 \times 150$ & 18 & $\mathrm{Bh}$ \\
\hline \multirow[t]{4}{*}{3} & House Roll Plate & $12 \mathrm{~mm} \times 650 \times 300$ & 2 & Sh \\
\hline & & $12 \mathrm{~mm} \times 300 \times 300$ & 2 & Sh \\
\hline & & $12 \mathrm{~mm} \times 110 \times 300$ & 4 & Sh \\
\hline & Roll bearing as & Dia. 280 & 2 & Set \\
\hline \multirow[t]{3}{*}{4} & House Roll Plate & $12 \mathrm{~mm} \times 1641 \times 180$ & 2 & Sh \\
\hline & Bracket & $12 \mathrm{~mm} 100 \times 550$ & 4 & Sh \\
\hline & Roll bearing as & Dia. 280 & 2 & Set \\
\hline \multirow[t]{3}{*}{5} & House Roll Plate & $12 \mathrm{~mm} \times 854 \times 180$ & 2 & Sh \\
\hline & & $12 \mathrm{~mm} \times 918 \times 150$ & 2 & Sh \\
\hline & Roll bearing as & Dia. 280 & 4 & Set \\
\hline \multirow[t]{3}{*}{6} & Bracket Karet & $12 \mathrm{~mm} \times 75 \times 175$ & 9 & Sh \\
\hline & Plat strip & $10 \mathrm{~mm} \times 34 \times 10000$ & 4 & Set \\
\hline & Rubber & $80 \times 40 \times 10000$ & 2 & Set \\
\hline \multirow[t]{6}{*}{7} & Wire rope & Dia. $16 \mathrm{~mm}$ & 100 & $M$ \\
\hline & Chain & Dia. $25 \mathrm{~mm}$ & 20 & $\mathrm{M}$ \\
\hline & Seal & Dia. $25 \mathrm{~mm}$ & 4 & $\mathrm{Bh}$ \\
\hline & Spanscrup & Dia. $25 \mathrm{~mm}$ & 2 & $\mathrm{Bh}$ \\
\hline & sch 80 pipe & Dia. 4" & 2 & Bar \\
\hline & Claim & Dia. $16 \mathrm{~mm}$ & 4 & Set \\
\hline \multirow[t]{3}{*}{8} & tonguelike & & & \\
\hline & Plate & $12 \mathrm{~mm} \times 1500 \times 6000$ & 1 & Sh \\
\hline & RB & Dia. 1" (@=12 m) & 13 & Bar \\
\hline
\end{tabular}

$\begin{array}{ll}\text { Brand } & \text { : Jiangsu Jiaoyan } \\ \text { Type } & : \text { JYR14 } \\ \text { Slewing Load } & : 14 \mathrm{KN} \\ \text { Suits } & : 14 \text { people }\end{array}$

\subsection{Winch and Electric Motor Power Ramp Door}

To calculate the winch and electric motor power, a list of materials used in the ramp door is required, the material list is obtained from secondary data collection, below is a list of materials that can be seen in table 3 , as follows:

Table 3. Rampdoor materials

First, by calculating the drum winch, based on the "Merchant Ship Design book 5 outfitting" as follows:

$$
\begin{aligned}
& l=\frac{k \times d \times L}{\pi \times N \times(D+n \times d)} ;(k w) \\
& l=\frac{1.2 \times 16.100000}{3 \times 14 \times 7 \times(280+7 \times 16)} ;(k w) \\
& l=222 \mathrm{~mm} \approx 230 \mathrm{~mm}
\end{aligned}
$$

Then perform the Hydraulic calculations, as follows: $M=F \times r ;(N m)$
$M=9810 \times 0.14$

$M=1373.4 \mathrm{Nm}$

Then calculate the flow rate of the electric motor, based on "Hydraulics. Theory and Applications, Bosch Rexroth p. 30", using the formula:

$Q=\frac{v \times n}{(n \times v \%) \cdot 10^{-1}} ;(l t / \mathrm{min})$

$Q=\frac{2.8 \times 913.4}{98 \times 10^{-1}} ;(l t / \mathrm{min})$

$Q=26.1 \mathrm{lt} / \mathrm{min}$

Then the calculation of the power input from the electric motor will be obtained using the formula:

$$
P=\frac{Q \cdot p}{6 \cdot t \%} ;(k w)
$$$$
P=\frac{144.12}{6.76 .5 \%} ;(k w)
$$

$P=65(k w)$

The last step is to calculate the power output of the electric motor using the formula:

$$
\begin{aligned}
P & =\frac{Q \cdot p \cdot t \%}{6 \times 10^{-4}} ;(k w) \\
P & =\frac{26 \cdot 207.76 .5 \%}{6 \times 10^{-4}} ;(k w) \\
P & =6.88(\mathrm{kw})
\end{aligned}
$$

Then we get a winch ramp door with the following capacities:

$\begin{array}{ll}\text { Brand } & : \text { Henan Mine Crane } \\ \text { Type } & : \text { JK2 } \\ \text { Rated speed } & : 24 \mathrm{~m} / \mathrm{min} \\ \text { Rope Capacity } & : 150 \mathrm{~m} \\ \text { Power } & : 7 \mathrm{KW} \\ \text { Weight } & : 550 \mathrm{~kg}\end{array}$

\subsection{Air Conditioner (AC)}

In calculating the electric motor power for the Air Conditioner (AC) it takes room volume data from the general arrangement of the LCT 1500 DWT ship which is obtained from secondary data collection. In this case, the measurement of the volume of the room contained on the main deck will be carried out, as follows:

Table 4. Volume and ac power requirement on main deck

\begin{tabular}{|c|c|c|l|c|}
\hline \multicolumn{5}{|c|}{ Main Deck } \\
\hline Room & Total & \multicolumn{2}{|c|}{ Volume } & $\begin{array}{c}\text { Power Needs } \\
\text { (KW) }\end{array}$ \\
\hline CO2 Rom & 1 & 51,8 & $\mathrm{~m} 3$ & 1,5 \\
\hline Provision Store & 1 & 70,6 & $\mathrm{~m} 3$ & 2,0 \\
\hline Crew 1 Room & 1 & 99,0 & $\mathrm{~m} 3$ & 2,8 \\
\hline Crew 2 Room & 1 & 99,0 & $\mathrm{~m} 3$ & 2,8 \\
\hline Crew 3 Room & 1 & 99,0 & $\mathrm{~m} 3$ & 2,8 \\
\hline Office & 1 & 85,8 & $\mathrm{~m} 3$ & 2,5 \\
\hline Mess Room & 1 & 303,7 & $\mathrm{~m} 3$ & 8,7 \\
\hline Galley & 1 & 109,9 & $\mathrm{~m} 3$ & 3,1 \\
\hline Total & & 918,7 & $\mathrm{~m} 3$ & 26,2 \\
\hline
\end{tabular}

In this case, the measurement of the volume of the room contained in the poop deck, as follows: 
Table 5. Volume and ac power requirement on poop deck

\begin{tabular}{|c|c|c|c|c|}
\hline \multicolumn{5}{|c|}{ Poop Deck } \\
\hline Room & Total & \multicolumn{2}{|c|}{ Volume } & $\begin{array}{c}\text { Power Needs } \\
(\mathbf{K W})\end{array}$ \\
\hline Captain Room & 1 & 105,3 & $\mathrm{~m} 3$ & 3,0 \\
\hline Crew 1 Room & 1 & 78,0 & $\mathrm{~m} 3$ & 2,2 \\
\hline Crew 1 Room & 1 & 76,3 & $\mathrm{~m} 3$ & 2,2 \\
\hline Owner Room & 1 & 69,3 & $\mathrm{~m} 3$ & 2,0 \\
\hline Engineering Room & 1 & 105,3 & $\mathrm{~m} 3$ & 3,0 \\
\hline \multicolumn{2}{|c|}{ Total } & 434,2 & $\mathrm{~m} 3$ & 12,4 \\
\hline
\end{tabular}

In this case, a measurement of the volume of the room contained in the wheel house deck as follows:

Table 6. Volume and ac power requirement on wheel house deck

\begin{tabular}{|c|c|c|c|c|}
\hline \multicolumn{5}{|c|}{ Wheel House Deck } \\
\hline Room & Total & \multicolumn{2}{|c|}{ Volume } & $\begin{array}{c}\text { Power Needs } \\
\text { (KW) }\end{array}$ \\
\hline Chart Space & 1 & 30,6 & $\mathrm{~m} 3$ & 0,9 \\
\hline $\begin{array}{c}\text { Radio \& Navigation } \\
\text { Space }\end{array}$ & 1 & 344,1 & $\mathrm{~m} 3$ & 9,8 \\
\hline \multicolumn{2}{|c|}{ Total } & 374,7 & $\mathrm{~m} 3$ & 10,7 \\
\hline
\end{tabular}

Then the total power requirement is obtained according to the calculation for the entire deck of $49.3 \mathrm{KW}$.

\subsection{Pumps}

The data on the power requirements of the pumps are obtained from secondary data collection, as follows:

Table 7. Pump power requirement

\begin{tabular}{|c|l|c|c|c|}
\hline \multirow{2}{*}{ NO } & \multicolumn{2}{|c|}{ ITEM } & No of & \multicolumn{2}{|c|}{ POWER } \\
\cline { 3 - 5 } & & set & output & input \\
\hline 1 & Bilge Pump / Fire Pump & 1 & 7,5 & 9 \\
\hline 2 & General Service \& Ballast Pump & 2 & 7,5 & 18 \\
\hline 3 & Sea Water Hydrophore Pump & 1 & 2,2 & 2,6 \\
\hline 4 & Fresh Water Hydrophore Pump & 1 & 2,2 & 2,6 \\
\hline 5 & Fuel Oil Transfer Pump & 1 & 1,5 & 1,8 \\
\hline 6 & Working Air Compressor & 1 & 1,5 & 1,8 \\
\hline 7 & Lub Oil Transfer Pump & 1 & 1,5 & 1,8 \\
\hline 8 & Fuel Oil Purifier & 1 & 2,2 & 2,6 \\
\hline 9 & Sludge Transfer Pump & 1 & 1,5 & 1,8 \\
\hline 10 & Sewage Treatment Plant & 1 & 2,5 & 3 \\
\hline 11 & Oil Water Separator & 1 & 0,4 & 0,5 \\
\hline
\end{tabular}

\subsection{Calculation of Lights on The Ship}

The planning of lighting on the ship is based on the book "Merchant Ship Handbook Vol 5", as follows:
Table 8. Ship navigation lightning

\begin{tabular}{|c|c|c|c|c|}
\hline No. & Item & $\begin{array}{c}\text { Power } \\
(\mathbf{K W})\end{array}$ & Jumlah & $\begin{array}{c}\text { Total Power } \\
(\mathbf{K W})\end{array}$ \\
\hline 1 & Main Light & 500 & 1 & 500 \\
\hline \multirow{2}{*}{2} & Side Light & & & \\
\cline { 2 - 5 } & a. Starboard Side & 50 & 1 & 50 \\
\cline { 2 - 5 } & b. port side & 50 & 1 & 50 \\
\hline 3 & Morse lamp & 100 & 1 & 100 \\
\hline \multirow{2}{*}{4} & Anchor Lamp & & & \\
\cline { 2 - 5 } & a. Bow Anchor Light & 40 & 2 & 80 \\
\cline { 2 - 5 } & b. Stern Anchor Light & 40 & 1 & 40 \\
\hline 5 & Stern Light & 75 & 1 & 75 \\
\hline 6 & Loading and Unloading Lights & 500 & 1 & 500 \\
\hline 7 & Sailing Lights & 40 & 1 & 40 \\
\hline 8 & Lifeboat Lamp & 75 & 1 & 75 \\
\hline 9 & Spotlights & 1000 & 1 & 1000 \\
\hline & & & & 2510 \\
\hline
\end{tabular}

Table 9. Poop deck lighting power requirements

\begin{tabular}{|c|c|c|c|c|c|c|}
\hline \multicolumn{7}{|c|}{ Poop Deck } \\
\hline \multirow{2}{*}{ No. } & \multirow{2}{*}{ Room } & \multirow{2}{*}{ Total } & \multicolumn{2}{|c|}{ Dimension (m) } & \multirow{2}{*}{$\mathrm{W} / \mathrm{m} 2$} & \multirow{2}{*}{$\begin{array}{c}\text { Power Needs } \\
\text { (KW) }\end{array}$} \\
\hline & & & $\overline{\mathbf{P}}$ & $\mathbf{L}$ & & \\
\hline 1 & Captain Room & 1 & 3,85 & 2,74 & 30 & 315,89 \\
\hline 2 & Crew 1 Room & 1 & 3,50 & 2,55 & 30 & 267,75 \\
\hline 3 & Crew 1 Room & 1 & 3,50 & 2,50 & 30 & 262,50 \\
\hline 4 & Owner Room & 1 & 3,50 & 2,55 & 30 & 267,75 \\
\hline 5 & Toilet 1 & 1 & 1,65 & 1,96 & 30 & 97,12 \\
\hline 6 & Toilet 2 & 1 & 1,50 & 1,50 & 30 & 67,50 \\
\hline 7 & Toilet 3 & 1 & 1,33 & 1,50 & 30 & 59,76 \\
\hline 8 & Toilet 4 & 1 & 1,65 & 1,96 & 30 & 97,12 \\
\hline 9 & Engineering Room & 1 & 3,85 & 2,74 & 30 & 315,89 \\
\hline \multicolumn{6}{|c|}{ Total } & 1751,28 \\
\hline
\end{tabular}

Then the planning for lighting power requirements on the wheel house deck is carried out based on table 11, as follows:

Table 10. Main deck lighting power requirements

\begin{tabular}{|c|c|c|c|c|c|c|}
\hline \multicolumn{7}{|c|}{ Main Deck } \\
\hline \multirow{2}{*}{ No. } & \multirow{2}{*}{ Room } & \multirow{2}{*}{ Total } & \multicolumn{2}{|c|}{ Dimension (m) } & \multirow{2}{*}{$\mathrm{W} / \mathrm{m} 2$} & \multirow{2}{*}{$\begin{array}{c}\text { Power Needs } \\
\text { (KW) }\end{array}$} \\
\hline & & & $\mathbf{P}$ & $\mathbf{L}$ & & \\
\hline 1 & CO2 Room & 1 & 1,5 & 3,54 & 30 & 159,08 \\
\hline 2 & Provision Store & 1 & 2,04 & 3,39 & 30 & 206,65 \\
\hline 3 & Toilet & 1 & 2,60 & 3,54 & 30 & 275,73 \\
\hline 4 & Crew 1 Room & 1 & 3,30 & 3,00 & 30 & 297,00 \\
\hline 5 & Crew 2 Room & 1 & 3,30 & 3,00 & 30 & 297,00 \\
\hline 6 & Crew 3 Room & 1 & 3,30 & 3,00 & 30 & 297,00 \\
\hline 7 & Office & 1 & 3,30 & 2,60 & 30 & 257,40 \\
\hline 8 & Mess Room & 1 & 6,60 & 4,80 & 30 & 950,40 \\
\hline 9 & Galley & 1 & 3,30 & 3,33 & 30 & 329,57 \\
\hline \multicolumn{6}{|c|}{ Total } & 3069,83 \\
\hline
\end{tabular}

Then do the lighting planning for the poop, the power requirements are obtained as follows:

Table 11. Wheel house deck lighting power requirements

\begin{tabular}{|c|c|c|c|c|c|c|}
\hline \multicolumn{7}{|c|}{ Wheel House Deck } \\
\cline { 4 - 5 } No. & \multirow{2}{*}{ Room } & Total & Dimension (m) & \multirow{2}{*}{$\begin{array}{c}\text { Power Needs } \\
\text { (KW) }\end{array}$} \\
\hline 1 & Chart Space & 1 & 1,584 & 1,93 & 30 & 91,86 \\
\hline 2 & $\begin{array}{c}\text { Radio \& Navigation } \\
\text { Space }\end{array}$ & 2 & 5,50 & 7,60 & 60 & 2508,00 \\
\hline \multicolumn{7}{|c|}{ Total } \\
\hline
\end{tabular}

There is an additional power requirement for internal lighting which can be seen in table 12 , as follows: 
Table 12. Other lighting power requirements

\begin{tabular}{|c|l|c|c|c|c|c|}
\hline \multicolumn{2}{|c|}{ Room } & Total & Dimension (m) & \multirow{2}{*}{ W/m2 } & $\begin{array}{c}\text { Power Needs } \\
\text { (KW) }\end{array}$ \\
\cline { 4 - 5 } & \multicolumn{1}{|c|}{ Total } & $\mathbf{P}$ & $\mathbf{L}$ & & 3691,80 \\
\hline 1 & Engine Room & 1 & 8,79 & 14 & 30 & 892,50 \\
\hline 2 & $\begin{array}{l}\text { Streering Gear } \\
\text { Room }\end{array}$ & 1 & 4,25 & 7 & 30 & 2400 \\
\hline 3 & $\begin{array}{l}\text { Aisle Lights Every } \\
\text { Deck }\end{array}$ & 60 & - & - & 40 & \\
\hline \multicolumn{7}{|c|}{} \\
\hline
\end{tabular}

Table 13. Power requirements for lighting outside the ship

\begin{tabular}{|c|c|c|}
\hline \multicolumn{3}{|c|}{ Lightning Outside S hip } \\
\hline No. & Item & Power Needs (KW) \\
\hline 1 & Outdoor Main Deck Alley & 1000 \\
\hline 2 & Poop Deck Outer Alley & 1000 \\
\hline 3 & Wheel House Deck Alley & 120 \\
\hline 4 & Lifeboat Launching & 1000 \\
\hline \multicolumn{2}{|c|}{ Total } \\
\hline
\end{tabular}

So,

$\Sigma=$ Total lights inside the ship + Total lights outside the ship + Total navigation lights

$=14405.27+3120+2510$

$=20035.27 \mathrm{Watt}$

$=20.04 \mathrm{~kW}$

It can be concluded that the need for lighting on the ship is $20.04 \mathrm{Kw}$

\subsection{Other Electrical Equipment Calculation}

The following are the power requirements of other installed electrical equipment, based on the book "Merchant Ship Handbook Vol 6", namely: In this case, the power used in kitchen equipment will be determined, as follows:

Table 14. Kitchen equipment power requirements

\begin{tabular}{|c|c|c|}
\hline \multicolumn{3}{|c|}{ Kitchen Equipment } \\
\hline No. & Item & $\begin{array}{c}\text { Power } \\
(\mathbf{K W})\end{array}$ \\
\hline 1 & Exhaust For Galley & 1,5 \\
\hline 2 & Waste Food Grinder & 0,75 \\
\hline 3 & Rice Cooker & 1,5 \\
\hline 4 & Freezer & 0,5 \\
\hline 5 & Water Boiler & 1,5 \\
\hline 6 & Electric cooking range & 25 \\
\hline 7 & Electric universal cooking machine & 0,75 \\
\hline 8 & Electric Coffee Burn & 0,1 \\
\hline 9 & Electric Fryer & 5 \\
\hline 10 & Refrigerator & 2,5 \\
\hline 11 & Baking Oven & 10 \\
\hline 12 & Hot Plate & 4 \\
\hline 13 & Soup Boiler & 9 \\
\hline 14 & Rice Washer & 0,4 \\
\hline 15 & Others & 77,5 \\
\hline & Total & \\
\hline
\end{tabular}

In this case, the power used in washing equipment will be determined which can be seen in table 15, as follows:
Table 15. Washing equipment power requirements

\begin{tabular}{|c|c|c|}
\hline \multicolumn{3}{|c|}{ Washing Equipment } \\
\hline No. & Item & Power (KW) \\
\hline 1 & Dishwasher & 0,5 \\
\hline 2 & Laundry $(2 \times 8 \mathrm{~kW})$ & 1,6 \\
\hline \multicolumn{2}{|c|}{ Total } & 2,1 \\
\hline
\end{tabular}

In this case, the determination of the power used in navigation and communication equipment can be seen in table 16 , as follows:

Table 16. Power requirements for navigation and communication equipment

\begin{tabular}{|c|c|c|}
\hline \multicolumn{3}{|c|}{ Navigation and Communication Equipment } \\
\hline No. & Item & Power (KW) \\
\hline 1 & Marine Radar & 0,5 \\
\hline 2 & Echo Sounder & 0,3 \\
\hline 3 & Radio & 0,3 \\
\hline 4 & Telephone & 0,08 \\
\hline 5 & GPS & 1 \\
\hline 6 & Telegraph & 0,08 \\
\hline 7 & Others & 3,5 \\
\hline & Total & 6 \\
\hline
\end{tabular}

In this case, the power used in other equipment will be determined which can be seen in table 17, as follows:

Table 17. Power requirements for other equipment

\begin{tabular}{|c|c|c|c|}
\hline \multicolumn{4}{|c|}{ Other Equipment } \\
\hline No. & Item & Total & Power (KW) \\
\hline 1 & Power pack for steering gear & 2 & 3,7 \\
\hline 2 & Power pack for bow thruster & 1 & 1,5 \\
\hline 3 & Exhaust fan for engine room & 2 & 7,5 \\
\hline 4 & Exhaust fan for emergency generator & 1 & 0,5 \\
\hline 5 & Battery Charger & 1 & 1 \\
\hline 6 & Exhaust fan for toilet & 5 & 1,5 \\
\hline \multicolumn{4}{|c|}{ Total } \\
\hline
\end{tabular}

\subsection{Analysis of Generator Power Capacity Calculation during the Day}

At this stage, calculations will be made during daytime conditions, as follows: 
Table 18. Harbour condition in day

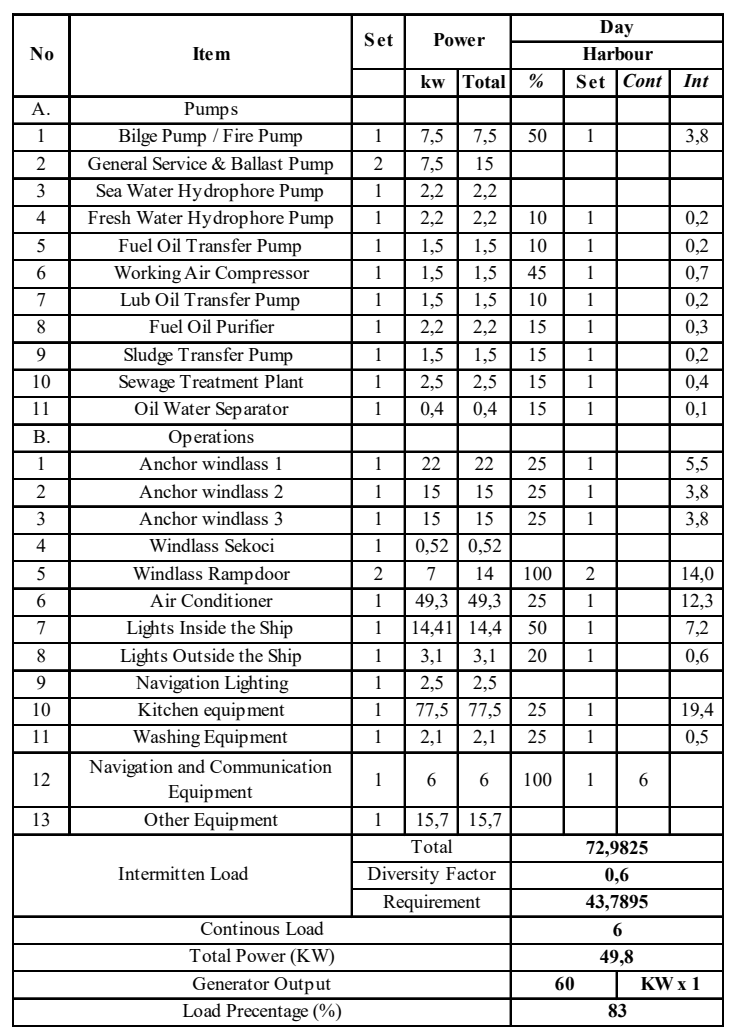

Table 19. Sailing condition in day

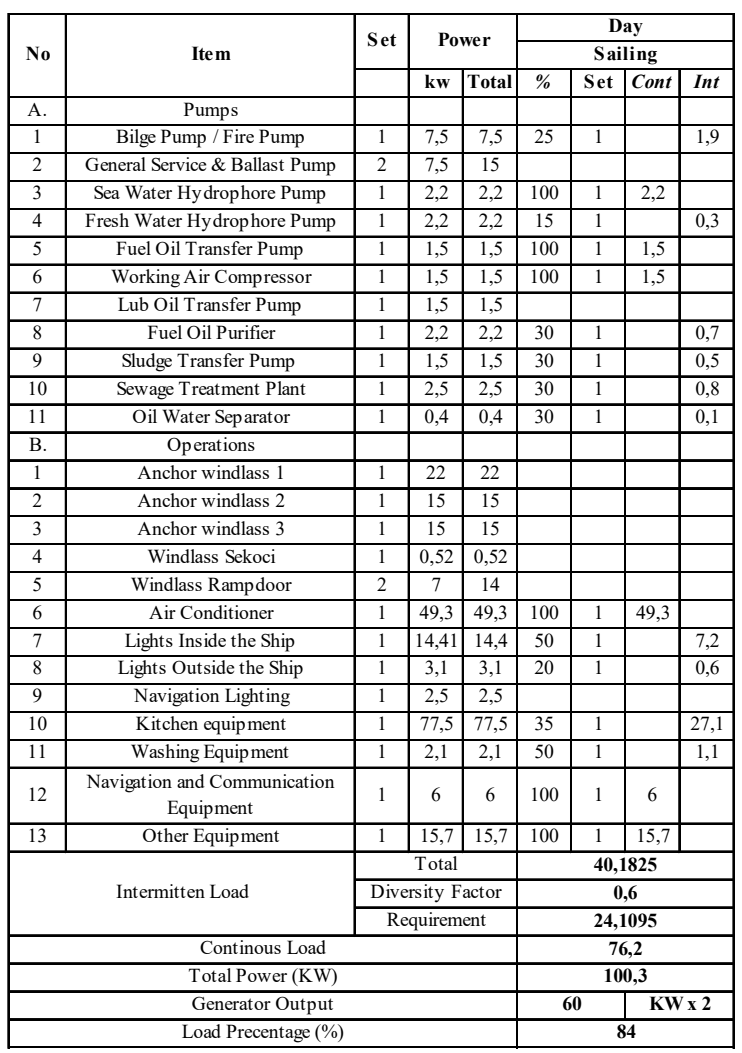

Table 20. Loading and unloading condition in day

\begin{tabular}{|c|c|c|c|c|c|c|c|c|}
\hline \multirow{3}{*}{ No } & \multirow{3}{*}{ Item } & \multirow{3}{*}{ Set } & \multirow{2}{*}{\multicolumn{2}{|c|}{ Power }} & \multicolumn{4}{|c|}{ Day } \\
\hline & & & & & \multicolumn{4}{|c|}{ Loading\&Unloading } \\
\hline & & & kw & Total & $\%$ & Set & Cont & Int \\
\hline A. & Pumps & & & & & & & \\
\hline 1 & Bilge Pump / Fire Pump & 1 & 7,5 & 7,5 & 25 & 1 & & 1,9 \\
\hline 2 & General Service \& Ballast Pump & 2 & 7,5 & 15 & 75 & 1 & & 5,6 \\
\hline 3 & Sea Water Hy drophore Pump & 1 & 2,2 & 2,2 & 25 & 1 & & 0,6 \\
\hline 4 & Fresh Water Hydrophore Pump & 1 & 2,2 & 2,2 & 10 & 1 & & 0,2 \\
\hline 5 & Fuel Oil Transfer Pump & 1 & 1,5 & 1,5 & 30 & 1 & & 0,5 \\
\hline 6 & Working Air Compressor & 1 & 1,5 & 1,5 & 10 & 1 & & 0,2 \\
\hline 7 & Lub Oil Transfer Pump & 1 & 1,5 & 1,5 & 10 & 1 & & 0,2 \\
\hline 8 & Fuel Oil Purifier & 1 & 2,2 & 2,2 & 25 & 1 & & 0,6 \\
\hline 9 & Sludge Transfer Pump & 1 & 1,5 & 1,5 & 25 & 1 & & 0,4 \\
\hline 10 & Sewage Treatment Plant & 1 & 2,5 & 2,5 & 25 & 1 & & 0,6 \\
\hline 11 & Oil Water Separator & 1 & 0,4 & 0,4 & 25 & 1 & & 0,1 \\
\hline B. & Operations & & & & & & & \\
\hline 1 & Anchor windlass 1 & 1 & 22 & 22 & 25 & 1 & & 5,5 \\
\hline 2 & Anchor windlass 2 & 1 & 15 & 15 & 25 & 1 & & 3,8 \\
\hline 3 & Anchor windlass 3 & 1 & 15 & 15 & 25 & 1 & & 3,8 \\
\hline 4 & Windlass Sekoci & 1 & 0,52 & 0,52 & & & & \\
\hline 5 & Windlass Rampdoor & 2 & 7 & 14 & 100 & 2 & 14 & \\
\hline 6 & Air Conditioner & 1 & 49,3 & 49,3 & 25 & 1 & & 12,3 \\
\hline 7 & Lights Inside the Ship & 1 & $\overline{14,41}$ & 14,4 & 50 & 1 & & $\overline{7,2}$ \\
\hline 8 & Lights Outside the Ship & 1 & 3,1 & 3,1 & 20 & 1 & & 0,6 \\
\hline 9 & Navigation Lighting & 1 & 2,5 & 2,5 & & & & \\
\hline 10 & Kitchen equipment & 1 & 77,5 & 77,5 & 25 & 1 & & 19,4 \\
\hline 11 & Washing Equip ment & 1 & 2,1 & 2,1 & 25 & 1 & & 0,5 \\
\hline 12 & $\begin{array}{c}\text { Navigation and Communication } \\
\text { Equipment }\end{array}$ & 1 & 6 & 6 & 100 & 1 & 6 & \\
\hline 13 & Other Equipment & 1 & 15,7 & 15,7 & & 1 & & \\
\hline \multirow{3}{*}{\multicolumn{2}{|c|}{ Intermitten Load }} & \multicolumn{3}{|c|}{ Total } & \multicolumn{4}{|c|}{63,7175} \\
\hline & & \multirow{2}{*}{\multicolumn{3}{|c|}{$\begin{array}{c}\text { Diversity Factor } \\
\text { Requirement }\end{array}$}} & \multicolumn{4}{|c|}{0,6} \\
\hline & & & & & \multicolumn{4}{|c|}{38,2305} \\
\hline \multicolumn{5}{|c|}{ Continous Load } & & & 20 & \\
\hline \multicolumn{5}{|c|}{ Total Power (KW) } & \multicolumn{4}{|c|}{58,2} \\
\hline \multicolumn{5}{|c|}{ Generator Output } & \multicolumn{4}{|c|}{ KW $\mathbf{2}$} \\
\hline \multicolumn{5}{|c|}{ Load Precentage (\%) } & \multicolumn{4}{|c|}{49} \\
\hline
\end{tabular}

Table 21. Emergency condition in day

\begin{tabular}{|c|c|c|c|c|c|c|c|c|}
\hline \multirow{3}{*}{ No } & \multirow{3}{*}{ Item } & \multirow{3}{*}{ Set } & \multirow{2}{*}{\multicolumn{2}{|c|}{ Power }} & \multirow{2}{*}{\multicolumn{4}{|c|}{$\begin{array}{c}\text { Day } \\
\text { Emergency }\end{array}$}} \\
\hline & & & & & & & & \\
\hline & & & kw & Total & $\%$ & Set & Cont & Int \\
\hline A. & $\begin{array}{l}\text { Pumps } \\
\text { Puts }\end{array}$ & & & & & & & \\
\hline 1 & Bilge Pump / Fire Pump & 1 & 7,5 & 7,5 & 100 & 1 & 7,5 & \\
\hline 2 & General Service \& Ballast Pump & 2 & 7,5 & 15 & 100 & 1 & & 7,5 \\
\hline 3 & Sea Water Hydrophore Pump & 1 & 2,2 & 2,2 & & & & \\
\hline 4 & Fresh Water Hydrop hore Pump & 1 & 2,2 & 2,2 & & & & \\
\hline 5 & Fuel Oil Transfer Pump & 1 & 1,5 & 1,5 & & & & \\
\hline 6 & Working Air Compressor & 1 & 1,5 & 1,5 & & & & \\
\hline 7 & Lub Oil Transfer Pump & 1 & 1,5 & 1,5 & & & & \\
\hline 8 & Fuel Oil Purifier & 1 & 2,2 & 2,2 & & & & \\
\hline 9 & Sludge Transfer Pump & 1 & 1,5 & 1,5 & & & & \\
\hline 10 & Sewage Treatment Plant & 1 & 2,5 & 2,5 & & & & \\
\hline 11 & Oil Water Separator & 1 & 0,4 & 0,4 & & & & \\
\hline B. & Operations & & & & & & & \\
\hline 1 & Anchor windlass 1 & 1 & 22 & 22 & & & & \\
\hline 2 & Anchor windlass 2 & 1 & 15 & 15 & & & & \\
\hline 3 & Anchor windlass 3 & 1 & 15 & 15 & & & & \\
\hline 4 & Windlass Sekoci & 1 & 0,52 & 0,52 & 100 & 1 & 0,5 & \\
\hline 5 & Windlass Rampdoor & 2 & 7 & 14 & & & & \\
\hline 6 & Air Conditioner & 1 & 49,3 & 49,3 & & & & \\
\hline 7 & Lights Inside the Ship & 1 & 14,41 & 14,4 & 100 & 1 & $\overline{14,4}$ & \\
\hline 8 & Lights Outside the Ship & 1 & 3,1 & 3,1 & & & & \\
\hline 9 & Navigation Lighting & 1 & 2,5 & 2,5 & & & & \\
\hline 10 & Kitchen equip ment & 1 & 77,5 & 77,5 & & & & \\
\hline 11 & Washing Equipment & 1 & 2,1 & 2,1 & & & & \\
\hline 12 & $\begin{array}{c}\text { Navigation and Communication } \\
\text { Equipment }\end{array}$ & 1 & 6 & 6 & 100 & 1 & 6,0 & \\
\hline 13 & Other Equipment & 1 & 15,7 & 15,7 & & & & \\
\hline \multirow{3}{*}{\multicolumn{2}{|c|}{ Intermitten Load }} & \multicolumn{3}{|c|}{ Total } & \multicolumn{4}{|c|}{7,5} \\
\hline & & \multicolumn{3}{|c|}{ Diversity Factor } & \multicolumn{4}{|c|}{0,6} \\
\hline & & \multicolumn{3}{|c|}{ Requirement } & \multicolumn{4}{|c|}{4,5} \\
\hline \multicolumn{5}{|c|}{ Continous Load } & & \multicolumn{3}{|c|}{28,4} \\
\hline \multirow{2}{*}{\multicolumn{5}{|c|}{$\begin{array}{l}\text { Total Power (KW) } \\
\text { Generator Output }\end{array}$}} & \multicolumn{4}{|c|}{32,9} \\
\hline & & & & & & 5 & & \\
\hline \multicolumn{5}{|c|}{ Load Precentage (\%) } & \\
\hline
\end{tabular}

power requirement of the ship during the day with a total intermittent load of $110.6 \mathrm{KW}$ and a total continuous load of $130.6 \mathrm{KW}$, as well as the power requirement in an emergency 
vessel condition of $32.9 \mathrm{KW}$ and the largest power is in the condition of the sailing vessel of $100,3 \mathrm{~kW}$.

\subsection{Analysis of Generator Power Capacity Calculation during Day Night}

At this stage, calculations will be made during day night conditions, as follows:

Table 22. Harbour condition in day night

\begin{tabular}{|c|c|c|c|c|c|c|c|c|}
\hline \multirow{3}{*}{ No } & \multirow{3}{*}{ Item } & \multirow{3}{*}{ Set } & \multirow{2}{*}{\multicolumn{2}{|c|}{ Power }} & \multirow{2}{*}{\multicolumn{4}{|c|}{$\begin{array}{c}\text { Night } \\
\text { Harbour }\end{array}$}} \\
\hline & & & & & & & & \\
\hline & & & kw & Total & $\%$ & Set & Cont & Int \\
\hline \multicolumn{9}{|c|}{ Pumps } \\
\hline 1 & Bilge Pump / Fire Pump & 1 & 7,5 & 7,5 & & & & \\
\hline 2 & General Service \& Ballast Pump & 2 & 7,5 & 15 & & & & \\
\hline 3 & Sea Water Hydrophore Pump & 1 & 2,2 & 2,2 & & & & \\
\hline 4 & Fresh Water Hy drophore Pump & 1 & 2,2 & 2,2 & 10 & 1 & & 0,2 \\
\hline 5 & Fuel Oil Transfer Pump & 1 & 1,5 & 1,5 & 10 & 1 & & 0,2 \\
\hline 6 & Working Air Compressor & 1 & 1,5 & 1,5 & 45 & 1 & & 0,7 \\
\hline 7 & Lub Oil Transfer Pump & 1 & 1,5 & 1,5 & 10 & 1 & & 0,2 \\
\hline 8 & Fuel Oil Purifier & 1 & 2,2 & 2,2 & 15 & 1 & & 0,3 \\
\hline 9 & Sludge Transfer Pump & 1 & 1,5 & 1,5 & 15 & 1 & & 0,2 \\
\hline 10 & Sewage Treatment Plant & 1 & 2,5 & 2,5 & 15 & 1 & & 0,4 \\
\hline 11 & Oil Water Sep arator & 1 & 0,4 & 0,4 & 15 & 1 & & 0,1 \\
\hline B. & \multicolumn{8}{|l|}{ Operations } \\
\hline 1 & Anchor windlass 1 & 1 & 22 & 22 & 25 & 1 & & 5,5 \\
\hline 2 & Anchor windlass 2 & 1 & 15 & 15 & 25 & 1 & & 3,8 \\
\hline 3 & Anchor windlass 3 & 1 & 15 & 15 & 25 & 1 & & 3,8 \\
\hline 4 & Windlass Sekoci & 1 & 0,52 & 0,52 & & & & \multirow[b]{2}{*}{14,0} \\
\hline 6 & Windlass Rampdoor & 2 & 7 & 14 & 100 & 2 & & \\
\hline 7 & Air Conditioner & 1 & 49,3 & 49,3 & 30 & 1 & & 14,8 \\
\hline 8 & Lights Inside the Ship & 1 & 14,41 & 14,4 & 100 & 1 & 14,4 & \\
\hline 9 & Lights Outside the Ship & 1 & 3,1 & 3,1 & 100 & 1 & 3,1 & \\
\hline 10 & Navigation Lighting & 1 & 2,5 & 2,5 & 100 & 1 & 2,5 & \multirow[b]{2}{*}{19,4} \\
\hline 11 & Kitchen equipment & 1 & 77,5 & 77,5 & 25 & 1 & & \\
\hline 12 & Washing Equipment & 1 & 2,1 & 2,1 & & 1 & & \\
\hline 13 & Navigation and Communication & 1 & 6 & 6 & 100 & 1 & 6 & \\
\hline 14 & Other Equipment & 1 & 15,7 & 15,7 & & 1 & & 0,0 \\
\hline \multirow{3}{*}{\multicolumn{2}{|c|}{ Intermitten Load }} & \multicolumn{3}{|c|}{ Total } & \multicolumn{4}{|c|}{63} \\
\hline & & \multicolumn{3}{|c|}{ Diversity Factor } & \multicolumn{4}{|c|}{0,6} \\
\hline & & & quirem & & \multicolumn{4}{|c|}{38,01} \\
\hline \multicolumn{5}{|c|}{ Continous Load } & \multicolumn{4}{|c|}{26,0} \\
\hline \multicolumn{5}{|c|}{ Total Power (KW) } & \multicolumn{4}{|c|}{64,0} \\
\hline \multicolumn{5}{|c|}{ Generator Output } & \multicolumn{4}{|c|}{ KW $\times 2$} \\
\hline \multicolumn{5}{|c|}{ Load Precentage (\%) } & & & 3 & \\
\hline
\end{tabular}

Table 23. Sailing condition in day night

\begin{tabular}{|c|c|c|c|c|c|c|c|c|}
\hline \multirow{2}{*}{ No } & \multirow{2}{*}{ Item } & \multirow{2}{*}{ Set } & \multicolumn{2}{|c|}{ Power } & \multicolumn{5}{|c|}{ Night } \\
\cline { 7 - 11 } & & & & & \multicolumn{5}{|c|}{ Sailing } \\
\hline & & & kw & Total & $\%$ & S et & Cont & Int \\
\hline A. & Pumps & & & & & & & \\
\hline 1 & Bilge Pump / Fire Pump & 1 & 7,5 & 7,5 & 25 & 1 & & 1,9 \\
\hline 2 & General Service \& Ballast Pump & 2 & 7,5 & 15 & & & & \\
\hline 3 & Sea Water Hydrophore Pump & 1 & 2,2 & 2,2 & 100 & 1 & 2,2 & \\
\hline 4 & Fresh Water Hydrophore Pump & 1 & 2,2 & 2,2 & 15 & 1 & & 0,3 \\
\hline 5 & Fuel Oil Transfer Pump & 1 & 1,5 & 1,5 & 100 & 1 & 1,5 & \\
\hline 6 & Working Air Compressor & 1 & 1,5 & 1,5 & 100 & 1 & 1,5 & \\
\hline 7 & Lub Oil Transfer Pump & 1 & 1,5 & 1,5 & & & & \\
\hline 8 & Fuel Oil Purifier & 1 & 2,2 & 2,2 & 30 & 1 & & 0,7 \\
\hline 9 & Sludge Transfer Pump & 1 & 1,5 & 1,5 & 30 & 1 & & 0,5 \\
\hline 10 & Sewage Treatment Plant & 1 & 2,5 & 2,5 & 30 & 1 & & 0,8 \\
\hline 11 & Oil Water Separator & 1 & 0,4 & 0,4 & 30 & 1 & & 0,1 \\
\hline B. & Operations & & & & & & & \\
\hline 1 & Anchor windlass 1 & 1 & 22 & 22 & & & & \\
\hline 2 & Anchor windlass 2 & 1 & 15 & 15 & & & & \\
\hline 3 & Anchor windlass 3 & 1 & 15 & 15 & & & & \\
\hline 4 & Windlass Sekoci & 1 & 0,52 & 0,52 & & & & \\
\hline 6 & Windlass Rampdoor & 2 & 7 & 14 & & & & \\
\hline 7 & Air Conditioner & 1 & 49,3 & 49,3 & 70 & 1 & 34,5 & \\
\hline 8 & Lights Inside the Ship & 1 & 14,41 & 14,4 & 100 & 1 & 14,4 & \\
\hline 9 & Lights Outside the Ship & 1 & 3,1 & 3,1 & 100 & 1 & 3,1 & \\
\hline 10 & Navigation Lighting & 1 & 2,5 & 2,5 & 100 & 1 & 2,5 & \\
\hline 11 & Kitchen equipment & 1 & 77,5 & 77,5 & 40 & 1 & & 31,0 \\
\hline
\end{tabular}

\begin{tabular}{|c|c|c|c|c|c|c|c|c|}
\hline 12 & Washing Equipment & 1 & 2,1 & 2,1 & & & & \\
\hline 13 & Navigation and Communication & 1 & 6 & 6 & 100 & 1 & 6,0 & \\
\hline 14 & Other Equipment & 1 & 15,7 & 15,7 & 100 & 1 & 15,7 & \\
\hline \multirow{3}{*}{\multicolumn{2}{|c|}{ Intermitten Load }} & \multicolumn{3}{|c|}{ Total } & \multicolumn{4}{|c|}{35,185} \\
\hline & & \multirow{2}{*}{\multicolumn{3}{|c|}{$\begin{array}{l}\text { Diversity Factor } \\
\text { Requiremententer }\end{array}$}} & \multicolumn{4}{|c|}{0,6} \\
\hline & & & & & & & & \\
\hline \multicolumn{5}{|c|}{ Continous Load } & \\
\hline \multicolumn{5}{|c|}{ Total Power (KW) } & \multicolumn{4}{|c|}{102,5} \\
\hline \multicolumn{5}{|c|}{ Generator Output } & \multirow{2}{*}{\multicolumn{4}{|c|}{$\cdots$}} \\
\hline & Load Precentage (\% & & & & & & & \\
\hline
\end{tabular}

Table 24. Loading and unloading condition in day night

\begin{tabular}{|c|c|c|c|c|c|c|c|c|}
\hline \multirow{3}{*}{ No } & \multirow{3}{*}{ Item } & \multirow{3}{*}{ Set } & \multirow{2}{*}{\multicolumn{2}{|c|}{ Power }} & \multicolumn{4}{|c|}{ Night } \\
\hline & & & & & \multicolumn{4}{|c|}{ Loading\&Unloading } \\
\hline & & & kw & Total & $\%$ & Set & Cont & Int \\
\hline A. & Pumps & & & & & & & \\
\hline 1 & Bilge Pump / Fire Pump & 1 & 7,5 & 7,5 & 25 & 1 & & 1,9 \\
\hline 2 & General Service \& Ballast Pump & 2 & 7,5 & 15 & 75 & 1 & & 5,6 \\
\hline 3 & Sea Water Hydrophore Pump & 1 & 2,2 & 2,2 & 25 & 1 & & 0,6 \\
\hline 4 & Fresh Water Hydrophore Pump & 1 & 2,2 & 2,2 & 10 & 1 & & 0,2 \\
\hline 5 & Fuel Oil Transfer Pump & 1 & 1,5 & 1,5 & 30 & 1 & & 0,5 \\
\hline 6 & Working Air Compressor & 1 & 1,5 & 1,5 & & & & \\
\hline 7 & Lub Oil Transfer Pump & 1 & 1,5 & 1,5 & 10 & 1 & & 0,2 \\
\hline 8 & Fuel Oil Purifier & 1 & 2,2 & 2,2 & 25 & 1 & & 0,6 \\
\hline 9 & Sludge Transfer Pump & 1 & 1,5 & 1,5 & 25 & 1 & & 0,4 \\
\hline 10 & Sewage Treatment Plant & 1 & 2,5 & 2,5 & 25 & 1 & & 0,6 \\
\hline 11 & Oil Water Separator & 1 & 0,4 & 0,4 & 25 & 1 & & 0,1 \\
\hline B. & Operations & & & & & & & \\
\hline 1 & Anchor windlass 1 & 1 & 22 & 22 & 25 & 1 & & 5,5 \\
\hline 2 & Anchor windlass 2 & 1 & 15 & 15 & 25 & 1 & & 3,8 \\
\hline 3 & Anchor windlass 3 & 1 & 15 & 15 & 25 & 1 & & 3,8 \\
\hline 4 & Windlass Sekoci & 1 & 0,52 & 0,52 & & & & \\
\hline 6 & Windlass Rampdoor & 2 & 7 & 14 & 100 & 2 & 14 & \\
\hline 7 & Air Conditioner & 1 & 49,3 & 49,3 & 30 & 1 & & 14,8 \\
\hline 8 & Lights Inside the Ship & 1 & 14,41 & 14,4 & 100 & 1 & 14,4 & \\
\hline 9 & Lights Outside the Ship & 1 & 3,1 & 3,1 & 100 & 1 & 3,1 & \\
\hline 10 & Navigation Lighting & 1 & 2,5 & 2,5 & 100 & 1 & 2,5 & \\
\hline 11 & Kitchen equipment & 1 & 77,5 & 77,5 & 25 & 1 & & 19,4 \\
\hline 12 & Washing Equipment & 1 & 2,1 & 2,1 & & & & \\
\hline 13 & Navigation and Communication & 1 & 6 & 6 & 100 & 1 & 6 & \\
\hline 14 & Other Equipment & 1 & 15,7 & 15,7 & & & & \\
\hline \multirow{3}{*}{\multicolumn{2}{|c|}{ Intermitten Load }} & \multicolumn{3}{|c|}{ Total } & \multicolumn{4}{|c|}{$\frac{1}{57,685}$} \\
\hline & & \multicolumn{3}{|c|}{ Diversity Factor } & \multicolumn{4}{|c|}{0,6} \\
\hline & & \multicolumn{3}{|c|}{ Requirement } & \multicolumn{4}{|c|}{34,611} \\
\hline \multicolumn{5}{|c|}{ Continous Load } & \multirow{2}{*}{\multicolumn{4}{|c|}{$\begin{array}{l}40,0 \\
74,6\end{array}$}} \\
\hline \multirow{2}{*}{\multicolumn{5}{|c|}{$\begin{array}{l}\text { Total Power (KW) } \\
\text { Generator Output }\end{array}$}} & & & & \\
\hline & & & & & 6 & & & $\overline{V \times 2}$ \\
\hline \multicolumn{5}{|c|}{ Load Precentage (\%) } & \multicolumn{4}{|c|}{62} \\
\hline
\end{tabular}

Table 25. Emergency condition in day night

\begin{tabular}{|c|c|c|c|c|c|c|c|c|}
\hline \multirow{2}{*}{ No } & \multirow{2}{*}{ Item } & \multirow{2}{*}{ Set } & \multicolumn{2}{|c|}{ Power } & \multicolumn{5}{|c|}{ Night } \\
\hline & & & & & \multicolumn{5}{|c|}{ Emergency } \\
\hline & & & kw & Total & $\%$ & Set & Cont & Int \\
\hline A. & Pumps & & & & & & & \\
\hline 1 & Bilge Pump / Fire Pump & 1 & 7,5 & 7,5 & 100 & 1 & 7,5 & \\
\hline 2 & General Service \& Ballast Pump & 2 & 7,5 & 15 & 100 & 1 & & 7,5 \\
\hline 3 & Sea Water Hydrophore Pump & 1 & 2,2 & 2,2 & & & & \\
\hline 4 & Fresh Water Hydrophore Pump & 1 & 2,2 & 2,2 & & & & \\
\hline 5 & Fuel Oil Transfer Pump & 1 & 1,5 & 1,5 & & & & \\
\hline 6 & Working Air Compressor & 1 & 1,5 & 1,5 & & & & \\
\hline 7 & Lub Oil Transfer Pump & 1 & 1,5 & 1,5 & & & & \\
\hline 8 & Fuel Oil Purifier & 1 & 2,2 & 2,2 & & & & \\
\hline 9 & Sludge Transfer Pump & 1 & 1,5 & 1,5 & & & & \\
\hline 10 & Sewage Treatment Plant & 1 & 2,5 & 2,5 & & & & \\
\hline 11 & Oil Water Separator & 1 & 0,4 & 0,4 & & & & \\
\hline B. & Operations & & & & & & & \\
\hline 1 & Anchor windlass 1 & 1 & 22 & 22 & & & & \\
\hline 2 & Anchor windlass 2 & 1 & 15 & 15 & & & & \\
\hline 3 & Anchor windlass 3 & 1 & 15 & 15 & & & & \\
\hline 4 & Windlass Sekoci & 1 & 0,52 & 0,52 & 100 & 1 & 0,5 & \\
\hline 6 & Windlass Rampdoor & 2 & 7 & 14 & & & & \\
\hline 7 & Air Conditioner & 1 & 49,3 & 49,3 & & & & \\
\hline 8 & Lights Inside the Ship & 1 & 14,41 & 14,4 & 100 & 1 & 14,4 & \\
\hline 9 & Lights Outside the Ship & 1 & 3,1 & 3,1 & 100 & 1 & 3,1 & \\
\hline 10 & Navigation Lighting & 1 & 2,5 & 2,5 & 100 & 1 & 2,5 & \\
\hline 11 & Kitchen equipment & 1 & 77,5 & 77,5 & & & & \\
\hline 12 & Washing Equipment & 1 & 2,1 & 2,1 & & & & \\
\hline
\end{tabular}




\begin{tabular}{|c|c|c|c|c|c|c|c|c|}
\hline 13 & Navigation and Communication & 1 & 6 & 6 & 100 & 1 & 6 & \\
\hline 14 & Other Equipment & 1 & 15,7 & 15,7 & & & & \\
\hline \multirow{3}{*}{\multicolumn{2}{|c|}{ Intermitten Load }} & \multicolumn{3}{|c|}{ Total } & \multicolumn{4}{|c|}{7,5} \\
\hline & & \multicolumn{3}{|c|}{ Diversity Factor } & \multicolumn{4}{|c|}{0,6} \\
\hline & & \multicolumn{3}{|c|}{ Requirement } & \multicolumn{4}{|c|}{4,5} \\
\hline \multicolumn{5}{|c|}{ Continous Load } & \multicolumn{4}{|c|}{34,0} \\
\hline \multicolumn{5}{|c|}{ Total Power (KW) } & \multicolumn{4}{|c|}{38,5} \\
\hline \multicolumn{5}{|c|}{ Generator Output } & \multicolumn{4}{|c|}{ KW X 1} \\
\hline \multicolumn{5}{|c|}{ Load Precentage (\%) } & \multicolumn{4}{|c|}{86} \\
\hline
\end{tabular}

it can be seen that the operational power requirement of the ship at night with a total intermittent load of 98,2 KW and a total continuous load of $181,5 \mathrm{KW}$ and power requirements in an emergency vessel condition of $38,5 \mathrm{KW}$ and the largest power is in the condition of the sailing vessel of $102,5 \mathrm{KW}$

\subsection{Generator Selection}

The generator selection is based on the calculation of the largest operational power capacity which is located on the condition of the ship sailing at night as much as 2 sets. Then we get a generator with the following capacity:

$\begin{array}{ll}\text { Brand } & \text { : Caterpillar } \\ \text { Type } & \text { : EM } 5153\end{array}$

Number of Cylinders : 4

Rpm/Frequency $:$ :1800/60 Hz

BHP $\quad: 60 \mathrm{KW}$

Bore $\quad: 105 \mathrm{~mm}$

Stroke $\quad: 127 \mathrm{~mm}$

Weight $\quad: 1142 \mathrm{~kg}$

Length $\quad: 1699 \mathrm{~mm}$

Width $\quad: 956 \mathrm{~mm}$

Height $\quad: 1245 \mathrm{~mm}$

Then the generator selection based on ship operations in an emergency is 1 set as follows:

Brand : John Deere

Type : 4045DFM70

Number of Cylinders : 4

Rpm/frequency $\quad: 1800 / 60 \mathrm{~Hz}$

BHP : $45 \mathrm{KW}$

Bore $\quad: 106.5 \mathrm{~mm}$

Stroke $\quad: 127 \mathrm{~mm}$

Weight $\quad: 462 \mathrm{~kg}$

Length $\quad: 885 \mathrm{~mm}$

Width $\quad: 712 \mathrm{~mm}$

Height $\quad: 902 \mathrm{~mm}$

\section{Conclusion}

In accordance with the calculation results obtained with various ship operating conditions. The condition of the ship anchored during the day is $49.8 \mathrm{KW}$. The condition of the ship anchored at night is $64 \mathrm{KW}$, The condition of the ship sailing during the day is $100.3 \mathrm{KW}$, The condition of the ship sailing at night is $102.5 \mathrm{KW}$. The condition of loading and unloading ships during the day is $58.2 \mathrm{KW}$. The condition of loading and unloading ships at night is $74.6 \mathrm{KW}$. Emergency ship condition during the day is $32.9 \mathrm{KW}$. The condition of the emergency ship at night is $38.5 \mathrm{KW}$. Determination of generator power capacity based on the operational electricity needs of the largest ship when the ship is sailing as many as 2 sets with power $60 \mathrm{KW} \times 2$. Then determine the generator power capacity based on the condition of the ship when in an emergency as much as 1 set with power $45 \mathrm{KW} \times 1$.

\section{References}

1. Rolan, ESH, UB, Optimization Analysis of Determination of Generator Power Capacity on MV Sinabung. Journal. Engineering. Shipping vol.8

2. Indonesian Classification Bureau (BKI), Rules for Electrical Installation Vo.4, Jakarta (2004)

3. Ricesno \& Reza, N, Calculation and Testing of Loads on Generators on Tugboat Hangtuah V, Sigma Teknika vol. 3, no.1 (2020)

4. Hamdana, R, Analysis of Declining Performance of Emergency Generators on Blackout Situations onboard MV. Shanti Indah, Marine Science Polytechnic, Semarang (2015)

5. Abdul, R \& Firdaus, Electrical Technical Analysis of the Swarna Putri Passenger Motor Boat, FTEKNIK Vol. 5, Issue 2, July 2018, p. 2 (2018)

6. Rinna, SS, RD, Electrical System on the Froschclass Navy Warship KRI Teluk Celukan Bawang 532', Journal of Energy \& Electricity vol. 9, no. 1, January (2017)

7. Sumardi \& Israr, Electrical System Analysis of 300 GTR Long Linear Tuna Fishing Vessel, University of Muhammadiyah, Makassar (2018)

8. Hadi, MS \& Arifai, M, Analysis of Voltage Frequency Stability of Electrical Power Systems PT. Aneka Tambang, Hasanuddin University, Makassar (2017)

9. Achmad, F, SS, IRK, Study of Technical Analysis of Passenger Ship Electrical Installation and Design Using Busbar Trunking, Journal of Engineering POMITS vol. 3, no. 1, 2014, p. 90 (2014)

10. Chris, JVS, Ship Electrical Installation, Hasanuddin University, Makassar (2017) 